\title{
Hyperspaces of two-dimensional continua
}

\author{
by
}

Michael Levin and Yaki Sternfeld (Haifa)

\begin{abstract}
Let $X$ be a compact metric space and let $\mathcal{C}(X)$ denote the space of subcontinua of $X$ with the Hausdorff metric. It is proved that every two-dimensional continuum $X$ contains, for every $n \geq 1$, a one-dimensional subcontinuum $T_{n}$ with $\operatorname{dim} \mathcal{C}\left(T_{n}\right) \geq n$. This implies that $X$ contains a compact one-dimensional subset $T$ with $\operatorname{dim} \mathcal{C}(T)=\infty$.
\end{abstract}

1. Introduction. Let $X$ be a compact metrizable space. $2^{X}$ denotes the space of closed subsets of $X$ endowed with the Hausdorff metric, and $\mathcal{C}(X)$ is the subset of $2^{X}$ which consists of the subcontinua of $X$. Both $2^{X}$ and $\mathcal{C}(X)$ are compact.

In [5] the authors proved that if $\operatorname{dim} X=2$ then $\operatorname{dim} \mathcal{C}(X)=\infty$. In this note we improve this result by showing that actually the 1-dimensional subcontinua of $X$ are responsible for the infinite dimensionality of $\mathcal{C}(X)$, more precisely: for every positive integer $n, X$ contains a one-dimensional subcontinuum $T_{n}$ with $\operatorname{dim} \mathcal{C}\left(T_{n}\right) \geq n$, and as a result, $X$ contains a onedimensional compact subset $T$ with $\operatorname{dim} \mathcal{C}(T)=\infty$. The following problem is still left open:

Question 1.1. Let $X$ be a 2-dimensional continuum. Does $X$ contain a 1-dimensional subcontinuum $T$ with $\operatorname{dim} \mathcal{C}(T)=\infty$ ?

In two extreme cases the answer is affirmative. It is proved in [6] that if $T$ is a 1-dimensional hereditarily indecomposable continuum then $\operatorname{dim} \mathcal{C}(T)$ is either 2 or $\infty$. Thus, if $X$ is a 2-dimensional hereditarily indecomposable continuum then the 1-dimensional continuum $T_{3} \subset X$ that we construct with $\operatorname{dim} \mathcal{C}\left(T_{3}\right) \geq 3$, actually satisfies $\operatorname{dim} \mathcal{C}\left(T_{3}\right)=\infty$ (see [3] for more information on hyperspaces of finite-dimensional hereditarily indecomposable continua). Note that this implies that every 3 -dimensional continuum $X$ contains a

1991 Mathematics Subject Classification: 54B20, 54F15, 54F45.

Key words and phrases: hyperspaces, hereditarily indecomposable continua, one- and two-dimensional continua. 
1-dimensional subcontinuum $T$ with $\operatorname{dim} \mathcal{C}(T)=\infty$ since by [1], $X$ contains a 2-dimensional hereditarily indecomposable continuum.

The hereditarily indecomposable continua are characterized by the property that their subcontinua do not intersect in a non-trivial manner (i.e. $A \cap B \neq \emptyset$ implies $A \subset B$ or $B \subset A$ ). If on the other hand a 2-dimensional continuum $X$ is rich with mutually intersecting 1-dimensional subcontinua (e.g. if $X$ is a Peano continuum or if $X$ is the product of two 1-dimensional continua) then again Question 1.1 has a positive answer for $X$.

We shall need the following result from [5] and include a short proof for it.

Theorem 1.2. Let $X$ be an $n$-dimensional compact metric space, $n<\infty$. There exists an n-dimensional hereditarily indecomposable continuum $Y$ and a light map $f$ of $Y$ into $X$.

Proof. We have $\operatorname{dim} X \times I=n+1, I=[0,1]$. By [1] there exists an $n$-dimensional hereditarily indecomposable continuum $Y \subset X \times I$. Let $P: X \times I \rightarrow X$ be the projection, and set $f=\left.P\right|_{Y}$. Then $f$ is light since a component of a fiber of $f$ is a subcontinuum of both $Y$ and $I$ and hence must be a singleton.

Recall that a map $W: \mathcal{C}(X) \rightarrow \mathbb{R}^{+}$is called a Whitney map if $W(\{x\})=0$ for all $x \in X$ and if $A \subset B, A \neq B$ in $\mathcal{C}(X)$ implies $W(A)<$ $W(B)$. Whitney maps always exist (see $[6]$ ).

Let $\psi: X \rightarrow Q$ be a map of compacta. Set $Q_{0}=\left\{z: z \in Q, \operatorname{dim} \psi^{-1}(z)\right.$ $\leq 0\}$ and $Q_{1}=Q \backslash Q_{0}=\left\{z \in Q: \operatorname{dim} \psi^{-1}(z) \geq 1\right\}$. We shall need the following result.

TheOREM 1.3. Let $X$ be an $n$-dimensional compact space, $n \geq 2$. There exist a 1-dimensional compactum $Q$ and a map $\psi: X \rightarrow Q$ such that $\operatorname{dim} \psi^{-1}\left(Q_{1}\right)=n-1$.

Proof. Let $Q$ be a dendrite with a dense set of nonseparating points. It is proved in Theorem 2.2 of [7] that for every compact space $X$ and every 0 -dimensional $\sigma$-compact subset $F$ of $X$, almost all maps $\psi \in C(X, Q)$ (i.e. all except a set of first category in the function space) satisfy $F \subset\{x \in X$ : $\left.\psi^{-1}(\psi(x))=\{x\}\right\}$, and thus $\psi^{-1}\left(Q_{1}\right) \subset X \backslash F$.

If $\operatorname{dim} X=n$ there exists a $\sigma$-compact 0-dimensional subset $F$ of $X$ such that $\operatorname{dim}(X \backslash F) \leq n-1$ ([7], Proposition 3.1). It follows that for almost all $\psi \in C(X, Q), \operatorname{dim} \psi^{-1}\left(Q_{1}\right)=n-1$ (note that $\operatorname{dim} \psi \geq n-1$ and hence $\left.\operatorname{dim} \psi^{-1}\left(Q_{1}\right) \geq n-1\right)$.

Another, more elementary proof of Theorem 1.3 can be obtained by applying the results of [2]. There Lelek constructs, for each $n \geq 2$, a map $f: I^{n} \rightarrow Q$, where $Q$ is a dendrite with $\operatorname{dim} f^{-1}\left(Q_{1}\right)=n-1$. (Lelek does not use the same terminology but it is easy to verify that $f$ indeed satisfies this.) 
Now, if $\operatorname{dim} X=n$, let $\varphi: X \rightarrow I^{n}$ be light; then for $\psi=f \circ \varphi: X \rightarrow Q$ we have $\operatorname{dim} \psi^{-1}\left(Q_{1}\right)=n-1$.

The general scheme of our note resembles that of [5] but it includes some additional ingredients and is more complicated.

\section{Proofs}

Theorem 2.1. Let $X$ be a 2-dimensional continuum and let $n$ be a positive integer. Then $X$ contains a 1-dimensional continuum $T_{n}$ with $\operatorname{dim} \mathcal{C}\left(T_{n}\right)>n$.

Corollary 2.2. Let $X$ be a 2-dimensional continuum. Then $X$ contains a 1-dimensional compact subset $T$ such that $\operatorname{dim} \mathcal{C}(T)=\infty$.

Proof. For each $n \geq 1$ let $X_{n}$ be a 2-dimensional continuum with $\operatorname{diam} X_{n} \leq 1 / n$ and $X_{1} \supset X_{2} \supset X_{3} \supset \ldots$ Let $T_{0}=\bigcap_{n=1}^{\infty} X_{n}\left(T_{0}\right.$ is a singleton) and by Theorem 2.1 let $T_{n} \subset X_{n}$ be a 1-dimensional continuum with $\operatorname{dim} \mathcal{C}\left(T_{n}\right)>n$. Take $T=\bigcup_{n=0}^{\infty} T_{n}$.

Lemma 2.3. Let $f: Y \rightarrow X$ be a light map of compacta. For every $\varepsilon>0$ there exist positive reals $\alpha(\varepsilon)$ and $\delta(\varepsilon)$ such that for every subset $B$ of $X$ with $\operatorname{diam} B \leq \delta(\varepsilon), f^{-1}(B)$ is decomposable as $f^{-1}(B)=\bigcup_{s=1}^{t} W^{s}$ with $\operatorname{diam} W^{s}<\varepsilon$ and $\operatorname{dist}\left(W^{s}, W^{r}\right) \geq \alpha(\varepsilon)$ for $s \neq r$. $\left(B y \operatorname{dist}\left(W^{s}, W^{r}\right)\right.$ we mean $\inf \left\{d\left(x^{s}, x^{r}\right): x^{s} \in W^{s}, x^{r} \in W^{r}\right\}$, where $d$ is a metric $)$.

Proof. Let $\varepsilon>0$. For $x \in X, \operatorname{dim} f^{-1}(x)=0$. Hence $f^{-1}(x)$ can be covered by a finite family $\mathcal{U}_{x}$ of open subsets of $Y$ with mesh $\mathcal{U}_{x}<\varepsilon$ and $\alpha(x)=\min \left\{\operatorname{dist}(A, B): A, B \in \mathcal{U}_{x}, A \neq B\right\}>0$. Let $V_{x}$ denote the union of the elements of $\mathcal{U}_{x} . V_{x}$ is a neighborhood of $f^{-1}(x)$ in $Y$. Let $W_{x}$ be an open neighborhood of $x$ in $X$ such that $f^{-1}\left(W_{x}\right) \subset V_{x}$. By compactness $X$ is covered by some $W_{x_{1}}, \ldots, W_{x_{n}}$. Let $\delta(\varepsilon)$ be the Lebesgue number of this cover; i.e. each subset $B$ of $X$ with $\operatorname{diam} B \leq \delta$ is contained in some $W_{x_{i}}$, and the lemma holds with $\alpha(\varepsilon)=\min \left\{\alpha\left(x_{i}\right): 1 \leq i \leq n\right\}$.

Lemma 2.4. Let $\mathcal{K} \subset \mathcal{C}(Y)$ be a decomposition of $Y$ which contains no singletons and which is closed in $\mathcal{C}(Y)$. Let $h: Y \rightarrow \mathcal{K}$ denote the corresponding (open) quotient map. Let $f$ be a light map of $Y$ into some continuum $X$, and let $g: Y \rightarrow \mathcal{C}(X)$ be defined by $g(y)=f(h(y))$. Then for every positive integer $n$ and every positive real $\varepsilon$ there exists a positive real $\alpha=\alpha(\varepsilon, n)$ such that for every closed subset $Y_{0} \subset Y$ with $\operatorname{dim} g\left(Y_{0}\right) \leq n$ there exist closed subsets $Z_{1}, \ldots, Z_{m}$ of $Y_{0}$ with $\operatorname{diam} Z_{i}<\varepsilon, 1 \leq i \leq m$ such that $\bigcup_{i=1}^{m} Z_{i}$ intersects every element of $\mathcal{K}$ which is contained in $Y_{0}$ and for $1 \leq i<j \leq m$ either $g\left(Z_{i}\right) \cap g\left(Z_{j}\right)=\emptyset$ or $\operatorname{dist}\left(Z_{i}, Z_{j}\right) \geq \varepsilon$. 
Pr o of. $h$ and $g$ are continuous since $\mathcal{K}$ is closed in $\mathcal{C}(Y)$. As $\mathcal{K}$ contains no singletons it follows that $\inf \{\operatorname{diam} K: K \in \mathcal{K}\}>0$; and since $f$ is light we see that $\inf \{\operatorname{diam} g(y): y \in Y\}=\inf \{\operatorname{diam} f(K): K \in \mathcal{K}\}=\lambda>0$.

As all $n$-dimensional spaces are embeddable in the same Euclidean space there exists an integer $N=N(n)$ such that for every $n$-dimensional compact space $H$ every open cover of $H$ has an open refinement $\left\{V_{1}, \ldots, V_{r}\right\}$ so that each $V_{i}$ intersects at most $N$ of the other $V_{j}$. Let $\varepsilon>0$ and $n$ be given. Let $\delta_{1}=\delta_{1}(\varepsilon)$ and $\alpha(\varepsilon)$ be as in Lemma 2.3.

Let $0<\delta=\min \left\{\delta_{1} / 2, \lambda /(6 N)\right\}$ (note that $\delta$ depends on $n$ and $\varepsilon$ ) and let $\alpha_{1}(\varepsilon, n)>0$ be small enough such that $d\left(y_{1}, y_{2}\right) \leq \alpha_{1}(\varepsilon, n)$ in $Y$ implies that $d\left(f\left(y_{1}\right), f\left(y_{2}\right)\right)<\delta($ in $X)$. Finally, let $\alpha(\varepsilon, n)=\min \left\{\alpha(\varepsilon), \alpha_{1}(\varepsilon, n)\right\}$.

Note that

(i) If $B_{1}, \ldots, B_{N}$ are $N$ subsets of $X$ with $\operatorname{diam} B_{i}<3 \delta$ then $\left\{B_{i}\right\}_{i=1}^{N}$ do not cover $g(y)$ for all $y \in Y$. Moreover, for every $y \in Y$ there exists a point $x \in g(y)$ such that $\operatorname{dist}\left(x, B_{i}\right) \geq 3 \delta$ for all $1 \leq i \leq N$. (Since $g(y)$ is a continuum of diameter $\geq \lambda$ and $\delta \leq \lambda /(6 N)$.)

Let $Y_{0} \subset Y$ be closed with $\operatorname{dim} g\left(Y_{0}\right) \leq n$. Let $\left\{\mathcal{V}_{1}, \ldots, \mathcal{V}_{r}\right\}$ be a closed cover of $g\left(Y_{0}\right)$ with mesh $<\delta$ (mesh with respect to the Hausdorff metric in $\mathcal{C}(X))$ such that each $\mathcal{V}_{i}$ intersects at most $N$ of the other $\mathcal{V}_{j}$. Then

(ii) For every $1 \leq i \leq r$, for every $A \in \mathcal{V}_{i}$, and every $x \in A, B(x, \delta)(=$ closed $\delta$-ball in $X$ with center at $x$ ) intersects every $B \in \mathcal{V}_{i}$ (since otherwise the Hausdorff distance between $A$ and $B$ would be more than $\delta$ ).

Now we construct inductively closed subsets $W_{1}, \ldots, W_{r}$ of $Y_{0}$ as follows: pick some $A_{1} \in \mathcal{V}_{1}$ and $x_{1} \in A_{1}$, and set $W_{1}=f^{-1}\left(B\left(x_{1}, \delta\right)\right) \cap g^{-1}\left(\mathcal{V}_{1}\right) \cap Y_{0}$. Assume that $W_{1}, \ldots, W_{j-1}$ were constructed as $W_{i}=f^{-1}\left(B\left(x_{i}, \delta\right)\right)$ $\cap g^{-1}\left(\mathcal{V}_{i}\right) \cap Y_{0}$ where $x_{i} \in A_{i} \in \mathcal{V}_{i}, 1 \leq i \leq j-1$. Let $A_{j} \in \mathcal{V}_{j}$. At most $N$ of $\mathcal{V}_{i}, 1 \leq i \leq j-1$, intersect $\mathcal{V}_{j}$. Assume these are $\mathcal{V}_{i_{1}}, \ldots, \mathcal{V}_{i_{N}}$. By (i) there exists a point $x_{j} \in A_{j}$ such that $\operatorname{dist}\left(x_{j}, B\left(x_{i_{l}}, 3 \delta\right)\right) \geq 3 \delta$ for all $1 \leq l \leq N$. Hence

(iii) $\operatorname{dist}\left(B\left(x_{j}, \delta\right), B\left(x_{i_{l}}, \delta\right)\right)>\delta$ for all $1 \leq l \leq N$

and we take $W_{j}=f^{-1}\left(B\left(x_{j}, \delta\right)\right) \cap g^{-1}\left(\mathcal{V}_{j}\right) \cap Y_{0}$. It follows from (ii) that $W_{i}$, $1 \leq i \leq r$, intersects every element of $\mathcal{K}$ which is contained in $Y_{0} \cap g^{-1}\left(\mathcal{V}_{i}\right)$ and so $\bigcup_{i=1}^{r} W_{i}$ intersects every element of $\mathcal{K}$ which is contained in $Y_{0}$.

From (iii) and the definition of $\alpha_{1}(\varepsilon, n)$ we obtain

(iv) for $1 \leq i<j \leq r$, if $g\left(W_{i}\right)=\mathcal{V}_{i}$ intersects $g\left(W_{j}\right)=\mathcal{V}_{j}$ then $\operatorname{dist}\left(B\left(x_{i}, \delta\right), B\left(x_{j}, \delta\right)\right)>\delta$ and hence $\operatorname{dist}\left(W_{i}, W_{j}\right) \geq \alpha_{1}(\varepsilon, n)$ (in $Y$ ) since $W_{i} \subset f^{-1}\left(B\left(x_{i}, \delta\right)\right)$.

As $\delta \leq \delta_{1} / 2$ and $W_{i} \subset f^{-1}\left(B\left(x_{i}, \delta\right)\right)$ we may apply Lemma 2.3 to decompose $W_{i}$ as $W_{i}=\bigcup_{s=1}^{t_{i}} W_{i}^{s}$ with $\operatorname{diam} W_{i}^{s}<\varepsilon$ and $\operatorname{dist}\left(W_{i}^{s_{1}}, W_{i}^{s_{2}}\right) \geq$ 
$\alpha(\varepsilon)$. For $1 \leq i<j \leq r$, if $g\left(W_{i}^{s}\right) \cap g\left(W_{j}^{t}\right) \neq \emptyset$ then by (iv), $\operatorname{dist}\left(W_{i}^{s}, W_{j}^{t}\right) \geq$ $\alpha_{1}(\varepsilon, n) \geq \alpha(\varepsilon, n)$ and we take $Z_{1}, \ldots, Z_{m}$ to be an enumeration of $\left\{W_{i}^{s}\right\}$, $1 \leq i \leq r, 1 \leq s \leq t_{i}$.

Proof of Theorem 2.1. Let $X$ be a 2-dimensional continuum. Apply Theorems 1.2 and 1.3 to find a 2-dimensional hereditarily indecomposable continuum $Y$ with a light map $f: Y \rightarrow X$, and a 1-dimensional continuum $Q$ with a map $\psi: X \rightarrow Q$ such that $\operatorname{dim} \psi^{-1}\left(Q_{1}\right)=1$. Let $\psi \circ f=\varphi \circ p$ denote the monotone light decomposition of the map $\psi \circ f: Y \rightarrow Q$ with $p: Y \rightarrow V=p(Y)$ monotone.

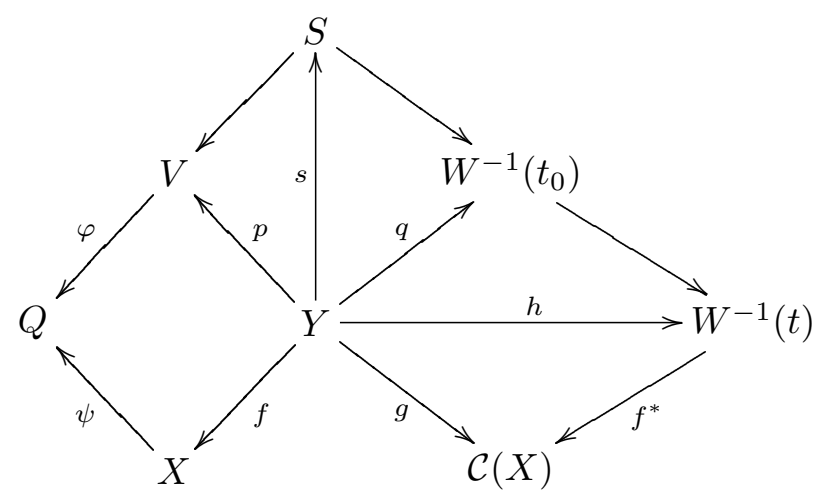

(The arrows not marked by letters in this diagram represent maps which exist, but are not referred to in the sequel.)

Let $F_{1}$ and $F_{2}$ be closed disjoint subsets of $Y$ such that

(i) every closed separator between $F_{1}$ and $F_{2}$ must have a component of diameter $\geq r=r\left(F_{1}, F_{2}\right)>0$.

Let $W: \mathcal{C}(Y) \rightarrow \mathbb{R}^{+}$be a Whitney map, and let $t>0$ be small enough such that

(ii) $\operatorname{mesh} W^{-1}(t)<r$.

$\mathcal{K}=W^{-1}(t)$ is a closed decomposition of $Y$ which contains no singletons. Let $h: Y \rightarrow W^{-1}(t)$ denote the quotient map. Let $n$ be a positive integer and set $\varepsilon=(1 / 3) \operatorname{dist}\left(F_{1}, F_{2}\right)>0$. Let $\alpha(\varepsilon, n)>0$ be the real obtained in Lemma 2.4. (Note that $g=f^{*} \circ h$, where $f^{*}: \mathcal{C}(Y) \rightarrow \mathcal{C}(X)$ is defined by $f^{*}(A)=f(A)$, i.e. $g(y)=f(h(y))$.)

Let $0<t_{0}<t$ be such that

(iii) $\operatorname{mesh} W^{-1}\left(t_{0}\right)<\min \{\alpha(\varepsilon, n), \varepsilon\}$.

Let $q: Y \rightarrow W^{-1}\left(t_{0}\right)$ be the quotient map. Then $q$ is an open monotone map with no trivial fibers. Let $s=p \wedge q$ denote the product of the maps $p$ and $q$, i.e. the fiber of $s$ at $y \in Y$ is the intersection of the fibers of $p$ and $q$ at $y$ (see [4]). Note that as $Y$ is hereditarily indecomposable and 
$p$ and $q$ are monotone, these fibers of $p$ and $q$ at $y$ actually contain one another. Thus, each fiber of $s$ is either a fiber of $p$ or of $q$. Let $S$ denote the range of $s$ and let $\mathcal{S}$ denote the decomposition of $Y$ induced by $s$. Set $Y_{q}=\left\{A: A \in \mathcal{S} \cap W^{-1}\left(t_{0}\right)\right\}$, i.e. $Y_{q}$ is the union of those fibers of $s$ which are fibers of $q$ (and thus are contained in some fiber of $p$ ).

$Y_{q}$ is closed in $Y$. To prove this we show that $\mathcal{S} \cap W^{-1}\left(t_{0}\right)$ is closed in $\mathcal{C}(Y)$. (Note that $\mathcal{S}$ may fail to be closed.) Let $\left\{A_{k}\right\}_{k=1}^{\infty} \subset \mathcal{S} \cap W^{-1}\left(t_{0}\right)$ converge to some $A \in \mathcal{C}(Y)$. Then $A \in W^{-1}\left(t_{0}\right)$ since $W^{-1}\left(t_{0}\right)$ is closed in $\mathcal{C}(Y)$. Each $A_{k}$ is contained in some fiber $B_{k}$ of $p$, and we may assume that $\left\{B_{k}\right\}$ converges in $\mathcal{C}(Y)$ to some continuum $B$. Clearly $A \subset B$ and as $p$ is continuous, $B$ is contained in some fiber of $p$. Hence $A$ is a fiber of $q$ and is contained in a fiber of $p$ so $A \in \mathcal{S}$ and $\mathcal{S} \cap W^{-1}\left(t_{0}\right)$ is closed.

We claim that

(iv) $\operatorname{dim} s\left(Y \backslash Y_{q}\right) \leq 1$.

Indeed, $Y \backslash Y_{q}$ is a union of fibers of $s$ which are also fibers of $p$ (but are not fibers of $q$ ). Hence the decomposition of $Y \backslash Y_{q}$ induced by the map $\left.s\right|_{Y \backslash Y_{q}}$ is identical to the decomposition induced by $\left.p\right|_{Y \backslash Y_{q}}$. Thus $s\left(Y \backslash Y_{q}\right)$ and $p\left(Y \backslash Y_{q}\right)$ are homeomorphic. It follows that $\operatorname{dim} s\left(Y \backslash Y_{q}\right)=\operatorname{dim} p\left(Y \backslash Y_{q}\right) \leq$ $\operatorname{dim} V$ and $\operatorname{dim} V \leq 1$ since $\varphi: V \rightarrow Q$ is light and $\operatorname{dim} Q=1$.

We also have $\operatorname{dim} f\left(Y_{q}\right)=1$. Indeed, let $A \in \mathcal{S} \cap W^{-1}\left(t_{0}\right)$. Then $A$ is a fiber of $q$ which is contained in a fiber $B$ of $p$. Moreover, $A$ is not a singleton and as $f$ is light both $f(A)$ and $f(B)$ are nontrivial continua in $X$. Recall that $\psi \circ f=\varphi \circ p$. Hence $\psi(f(B))=\varphi(p(B))$ and as $B$ is a fiber of $p$, $\varphi(p(B))$ is a singleton and $\psi$ is constant on $f(B)$. It follows that $f(B)$ is contained in $\psi^{-1}\left(Q_{1}\right)$ (which is the union of all fibers of $\psi$ with dimension $>0)$ and also that $f\left(Y_{q}\right) \subset \psi^{-1}\left(Q_{1}\right)$ and as $\operatorname{dim} \psi^{-1}\left(Q_{1}\right) \leq 1$, we have $\operatorname{dim} f\left(Y_{q}\right) \leq 1$.

Set $Y_{0}=\bigcup\left\{E: E \in W^{-1}(t), E \subset Y_{q}\right\}$. Thus $Y_{0}$ consists of those fibers of $h$ which are contained in $Y_{q}$. Note that the decomposition $W^{-1}\left(t_{0}\right)$ strictly refines $W^{-1}(t)$, so if $E \in W^{-1}(t)$ then $E$ is a union of fibers of $q$.

(v) $Y_{0}$ is closed in $Y$

since $\mathcal{D}=\left\{E: E \in W^{-1}(t), E \subset Y_{q}\right\}$ is closed in $\mathcal{C}(Y)$. The latter holds since if $E_{k} \in \mathcal{D}$ and $E_{k} \rightarrow E$ in $\mathcal{C}(Y)$ then $E \in W^{-1}(t)$ and $E \subset Y_{q}$ as $W^{-1}(t)$ is closed in $\mathcal{C}(Y)$ and $Y_{q}$ is closed in $Y$.

And as $f\left(Y_{0}\right) \subset f\left(Y_{q}\right)$ we also have

(vi) $\operatorname{dim} f\left(Y_{0}\right) \leq 1$.

(Note that as $f$ is light, $\operatorname{dim} Y_{q} \leq 1$ too.)

We claim that $\operatorname{dim} g\left(Y_{0}\right)>n$. Once we show this we are done. Indeed, $g\left(Y_{0}\right)=\left\{f(h(y)): y \in Y_{0}\right\}$. For $y \in Y_{0}, h(y) \in W^{-1}(t)$ is contained in $Y_{0}$ 
and it follows that $g\left(Y_{0}\right) \subset \mathcal{C}\left(f\left(Y_{0}\right)\right)$. This implies that $\operatorname{dim} \mathcal{C}\left(f\left(Y_{0}\right)\right)>n$. Hence $f\left(Y_{0}\right)$ (which is compact by (v) and 1-dimensional by (vi)) must contain a 1-dimensional component $T_{n}$ with $\operatorname{dim} \mathcal{C}\left(T_{n}\right)>n$.

Aiming at a contradiction assume $\operatorname{dim} g\left(Y_{0}\right) \leq n$. Then we may apply Lemma 2.4. Let $Z_{1}, \ldots, Z_{m} \subset Y_{0}$ be from the conclusion of Lemma 2.4 for $\mathcal{K}=W^{-1}(t)$. Then

(vii) the sets $s\left(Z_{i}\right), 1 \leq i \leq m$, are mutually disjoint.

Indeed, the map $s$ is a factor of $g$. By this we mean that the fibers of $s$ are contained in those of $g$. Hence $g\left(Z_{i}\right) \cap g\left(Z_{j}\right)=\emptyset$ implies $s\left(Z_{i}\right) \cap s\left(Z_{j}\right)=\emptyset$. If for some $i<j, g\left(Z_{i}\right) \cap g\left(Z_{j}\right) \neq \emptyset$ then by Lemma 2.4, $\operatorname{dist}\left(Z_{i}, Z_{j}\right) \geq$ $\alpha(\varepsilon, n)$. By (iii) each fiber of $q$ has diameter $<\alpha(\varepsilon, n)$, which implies that $q\left(Z_{i}\right) \cap q\left(Z_{j}\right)=\emptyset$ and as $s$ is a factor of $q$ too, $s\left(Z_{i}\right) \cap s\left(Z_{j}\right)=\emptyset$.

(viii) $s\left(F_{1}\right) \cap s\left(F_{2}\right)=\emptyset$.

This holds since $q$ and hence $s$ are $\varepsilon$-maps (by (iii)) and $\varepsilon=\frac{1}{3} \operatorname{dist}\left(F_{1}, F_{2}\right)$. The same argument combined with the fact that $\operatorname{diam} Z_{i}<\varepsilon$ also implies that

(ix) for every $1 \leq i \leq m, s\left(Z_{i}\right)$ intersects at most one of the sets $s\left(F_{1}\right)$ and $s\left(F_{2}\right)$.

Set $H_{1}=s\left(F_{1}\right) \cup\left(\bigcup\left\{s\left(Z_{i}\right): s\left(F_{1}\right) \cap s\left(Z_{i}\right) \neq \emptyset\right\}\right)$ and $H_{2}=s\left(F_{2}\right) \cup$ $\left(\bigcup\left\{s\left(Z_{i}\right): s\left(F_{1}\right) \cap s\left(Z_{i}\right)=\emptyset\right\}\right)$. By (ix), $H_{1} \cap H_{2}=\emptyset$. By (iv), $\operatorname{dim} s\left(Y \backslash Y_{q}\right)$ $\leq 1$ hence there exists a closed subset $L$ of $S=s(Y)$ which separates between $H_{1}$ and $H_{2}$ in $S$ such that $\operatorname{dim} L \cap s\left(Y \backslash Y_{q}\right)=0$. Then $L$ also separates $s\left(F_{1}\right)$ from $s\left(F_{2}\right)$ and

(x) $L \cap s\left(\bigcup_{i=1}^{m} Z_{i}\right)=\emptyset$. $=\emptyset$.

By (i), $s^{-1}(L)$ has a component $M$ with $\operatorname{diam} M>r$. Then $M \cap\left(Y \backslash Y_{q}\right)$

Indeed, by (ii) fibers of $s$ have diameter $<r$. Hence $s(M)$ is a nontrivial continuum in $L$. If $y \in M \cap\left(Y \backslash Y_{q}\right)$ then $w=s(y) \in L \cap s\left(Y \backslash Y_{q}\right)$. Since $Y_{q}$ is a union of fibers of $s$ (those fibers which are also fibers of $q$ ) we have $s\left(Y \backslash Y_{q}\right)=s(Y) \backslash s\left(Y_{q}\right)$ and hence $w \in L \backslash s\left(Y_{q}\right)=L \cap s\left(Y \backslash Y_{q}\right)$. As $s\left(Y_{q}\right)$ is closed and $\operatorname{dim} L \backslash s\left(Y_{q}\right)=\operatorname{dim} L \cap s\left(Y \backslash Y_{q}\right)=0,\{w\}$ is a component of $L$ and hence $s(M) \subset\{w\}$, which contradicts the fact that $s(M)$ is nontrivial.

It follows that $M \subset Y_{q}$. Let $A \in W^{-1}(t)$ be such that $A \cap M \neq \emptyset$. As $\operatorname{diam} A<r$, we have $A \subset M \subset s^{-1}(L)$ (by (ii)). So $A \subset Y_{0}$ and $s(A) \subset L$. By Lemma 2.4, $\bigcup_{i=1}^{m} Z_{i}$ intersects $A$ and hence $s\left(\bigcup_{i=1}^{m} Z_{i}\right)$ intersects $L$, contradicting $(\mathrm{x})$. This contradiction implies $\operatorname{dim} g\left(Y_{0}\right)>n$ and concludes the proof. 


\section{References}

[1] R. H. Bing, Higher-dimensional hereditarily indecomposable continua, Trans. Amer. Math. Soc. 71 (1951), 267-273.

[2] A. Lelek, On mappings that change dimension of spheres, Colloq. Math. 10 (1963), $45-48$.

[3] M. Levin, Hyperspaces and open monotone maps of hereditarily indecomposable continua, Proc. Amer. Math. Soc., to appear.

[4] M. Levin and Y. Sternfeld, Mappings which are stable with respect to the property $\operatorname{dim} f(X) \geq k$, Topology Appl. 52 (1993), 241-265.

[5] - - - The space of subcontinua of a 2-dimensional continuum is infinite dimensional, Proc. Amer. Math. Soc., to appear.

[6] S. B. Nadler Jr., Hyperspaces of Sets, Dekker, 1978.

[7] Y. Sternfeld, Mappings in dendrites and dimension, Houston J. Math. 19 (1993), 483-497.

Department of Mathematics

Haifa University

Mount Carmel

Haifa 31905, Israel

E-mail: levin@mathcs2.haifa.ac.il yaki@mathcs2.haifa.ac.il

Received 2 June 1995;

in revised form 5 December 1995 